\title{
Impact of methicillin resistance on mortality in Staphylococcus aureus VAP: a systematic review
}

\author{
Z. Athanassa*, I.I. Siempos* and M.E. Falagas*,\#,9
}

ABSTRACT: The aim of the present study was to estimate the impact of methicillin resistance on mortality in ventilator-associated pneumonia (VAP) due to Staphylococcus aureus.

PubMed, Scopus and the bibliographies of the eligible studies were searched. The DerSimonian-Laird random effects model was used to determine the effect of methicillin resistance on mortality.

Eight articles were included. Crude in-hospital mortality was higher in patients with VAP due to methicillin-resistant $S$. aureus (MRSA) than in those with VAP due to methicillin-sensitive S. aureus (MSSA). This was also the case for crude intensive care unit mortality. However, three of the selected studies, which adjusted for potential confounding factors, including adequacy of empirical treatment and severity of illness, demonstrated no difference in in-hospital mortality between patients with MRSA and MSSA VAP. This was not the case for another eligible study that also made adjustment, but for confounders other than those shown above.

The limited available evidence seems to suggest that methicillin resistance is associated with death among persons acquiring Staphylococcus aureus ventilator-associated pneumonia. However, although supported by even more limited data, adjustment for risk factors suggests that this association may not be causal, but probably due to confounders, such as the adequacy of empirical treatment and severity of illness.

KEYWORDS: Antimicrobial resistance, critical illness, healthcare-associated pneumonia, morbidity, nosocomial pneumonia, survival

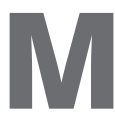

ost experts appear to agree that infections due to drug-resistant pathogens result in poorer clinical and economic outcomes as opposed to those due to sensitive strains [1-3]. With regard to Staphylococcus aureus infections, this opinion relies mainly on the findings of a recent meta-analysis of studies published during 1980-2000 comparing the mortality of patients with methicillin-resistant S. aureus (MRSA) and methicillin-sensitive $S$. aureus (MSSA) bacteraemia [2]. By accumulating data from 31 studies, this analysis revealed that mortality was higher in patients with MRSA bacteraemia than in those with MSSA bacteraemia, with a pooled odds ratio (OR) of 1.93 (95\% confidence interval (CI) 1.54-2.42) [2]. Interestingly, a number of more recent studies have confirmed these findings $[4,5]$.

However, methodological flaws in the studies included in the above meta-analysis [2] may limit its conclusions. Indeed, the authors of the analysis clearly stated that the individual studies did not provide specific data regarding the adequacy of the initially administered antimicrobial regimen, which probably confounds the relationship between methicillin resistance and fatality [6, 7]. In addition, they considered as questionable the quality of adjustment for severity of illness of bacteraemic patients in the selected studies, mainly because of the lack of a validated method for this purpose. Thus scepticism remains as to whether methicillin resistance influences patient outcome, a scepticism further enhanced by the paucity, to date, of investigations establishing a definitive association between antimicrobial resistance and $S$. aureus virulence $[8,9]$.

The impact of methicillin resistance on mortality has also been addressed in patients with $S$. aureus ventilator-associated pneumonia (VAP). Studies exploring this issue have produced conflicting results, thereby further fuelling the controversy. $\mathrm{VAP}$ is the most common infection in the intensive care unit (ICU) setting, accounting for

\section{AFFILIATIONS}

*Alfa Institute of Biomedical Sciences,

"Dept of Medicine, Henry Dunant Hospital, Athens, Greece.

"Dept of Medicine, Tufts University School of Medicine, Boston, MA, USA.

CORRESPONDENCE

M.E. Falagas

Alfa Institute of Biomedical Sciences 9 Neapoleos Street

15123 Marousi

Greece

Fax: 302106839605

E-mail: m.falagas@aibs.gr

Received:

July 022007

Accepted after revision:

October 172007

STATEMENT OF INTEREST None declared.

European Respiratory Journal Print ISSN 0903-1936 Online ISSN 1399-3003 
almost a third of total nosocomial infections [10, 11]. S. aureus has been recognised as one of the most common pathogens isolated in patients with VAP, and the prevalence of methicillin-resistant strains is increasing [12]. Thus elucidation of the impact of resistance on patient outcome may be essential in defining the prognosis of the individual patient with $S$. aureus VAP.

Thus attempts were made to exploit the available evidence in order to quantify the effect, if any, of methicillin resistance on mortality in patients with VAP due to $S$. aureus. A systematic review and analysis of the accumulated data were performed for this purpose.

\section{METHODS}

\section{Data sources}

A systematic literature search of PubMed and Scopus was conducted (during February 2007) to trace relevant studies. The search terms used were: ("ventilator-associated pneumonia" OR "ventilator associated pneumonia" OR "nosocomial pneumonia" AND "methicillin". The search was also repeated using the term "oxacillin" instead of "methicillin". In an attempt to increase completeness, the bibliographies of the articles initially retrieved were also hand-searched. Abstracts of conference proceedings were not sought and no restriction was set on publication date or language.

\section{Study selection}

The literature search was performed independently by two investigators (Z. Athanassa and I.I. Siempos). Both observational and interventional studies were considered eligible if they provided data on the mortality of patients with MRSA and MSSA VAP. Reports investigating nosocomial pneumonia were included in the present analysis only if $\geqslant 75 \%$ of the study population had received mechanical ventilation.

\section{Data extraction}

The studies were independently reviewed by two investigators (Z. Athanassa and I.I. Siempos). The following data were extracted: first author, year of publication, country, study design, type of ICU, study population, number of patients with S. aureus VAP, and types of culture used for confirmation of the $S$. aureus VAP diagnosis. In addition, data were collected on the number of patients, mortality and length of ICU stay after VAP onset in the MRSA and MSSA VAP groups. Factors thought to act as potential confounders (mean age, sex, underlying diseases, severity of illness on ICU admission, adequacy of empirical treatment, length of hospital stay and mechanical ventilation before VAP onset, presence of bacteraemia and presence of an outbreak of MRSA infection) were also recorded [2]. Finally, information dealing with adjusted mortality, methods of adjustment and factors for which mortality had been adjusted were gathered.

\section{Definitions}

Outcomes of the systematic review

Crude in-hospital mortality was the main outcome of the present systematic review. Adjusted in-hospital mortality and crude ICU mortality, as well as length of ICU stay after VAP onset, in patients with MRSA and MSSA VAP also served as outcomes of the present study.

\section{Ventilator-associated pneumonia}

Pneumonia was defined by clinical, laboratory and imaging findings attributed by the authors of the individual selected articles to this infection. When the above findings occurred in patients who had received mechanical ventilation for $\geqslant 48 \mathrm{~h}$, the pneumonia was regarded as ventilator-associated.

\section{Data analysis and statistical methods}

The number of MRSA (versus MSSA) VAP patients who died was divided by the total number of MRSA (versus MSSA) VAP patients. Pooled ORs and 95\% CIs were calculated using a conservative DerSimonian-Laird random effects model. Heterogeneity among the results of the included studies was tested using the Chi-squared test; a p-value $<0.10$ was defined as denoting significance in the analysis of heterogeneity.

\section{RESULTS}

\section{Selected studies}

The selection process applied to capture the studies included in the present systematic review is depicted in figure 1. The PubMed search yielded 246 potentially relevant reports; 239 of them were excluded for the reasons detailed in figure 1 . Two studies $[13,14]$ enrolled, in large proportion, the same study population (i.e. patients included in the PneumA trial, a multicentric randomised controlled trial comparing 8 versus 15 days of antimicrobial treatment for VAP [15]); thus, one [13] of these two studies was omitted. Three additional potentially relevant reports were found by hand-searching the references of the articles initially retrieved. Two $[16,17]$ of these three studies were excluded because $<75 \%$ of the patients enrolled had received mechanical ventilation [16] or because they did not provide specific data on MRSA VAP [17]. Search employing the Scopus database did not reveal any additional reports fulfilling the present inclusion criteria. This was also the case for the search carried out replacing the term "methicillin" with "oxacillin". Thus eight articles [14, 18-24] were included in the present systematic review.

\section{Characteristics of the selected studies}

The characteristics of the eight selected articles [14, 18-24], representing 2,814 patients with $S$. aureus VAP, are listed in table 1 . Their mean (range) cohort size was 154 patients (491,851). All were designed as prospective (four out of eight; $50 \%)[19,22-24]$ or retrospective $(50 \%)$ observational studies $[14,18,20,21]$. One [14] of the included studies was partly based on the 401 patients included in the previously mentioned PneumA trial [15].

The VAP diagnosis was assessed using clinical, laboratory and imaging findings in three $[18,21,24]$ of the eight eligible reports. In contrast, in the remaining five studies [14, 19, 20, 22, 23], VAP diagnostic criteria included microbiological confirmation by qualitative or quantitative culture of respiratory specimens (i.e. protected brush or catheter specimen or bronchoalveolar lavage fluid) and/or blood (with the same microorganism isolated from blood and sputum specimens).

\section{Study population}

The characteristics of the patients of the studies included in the systematic review are depicted in table 2. Compared with 


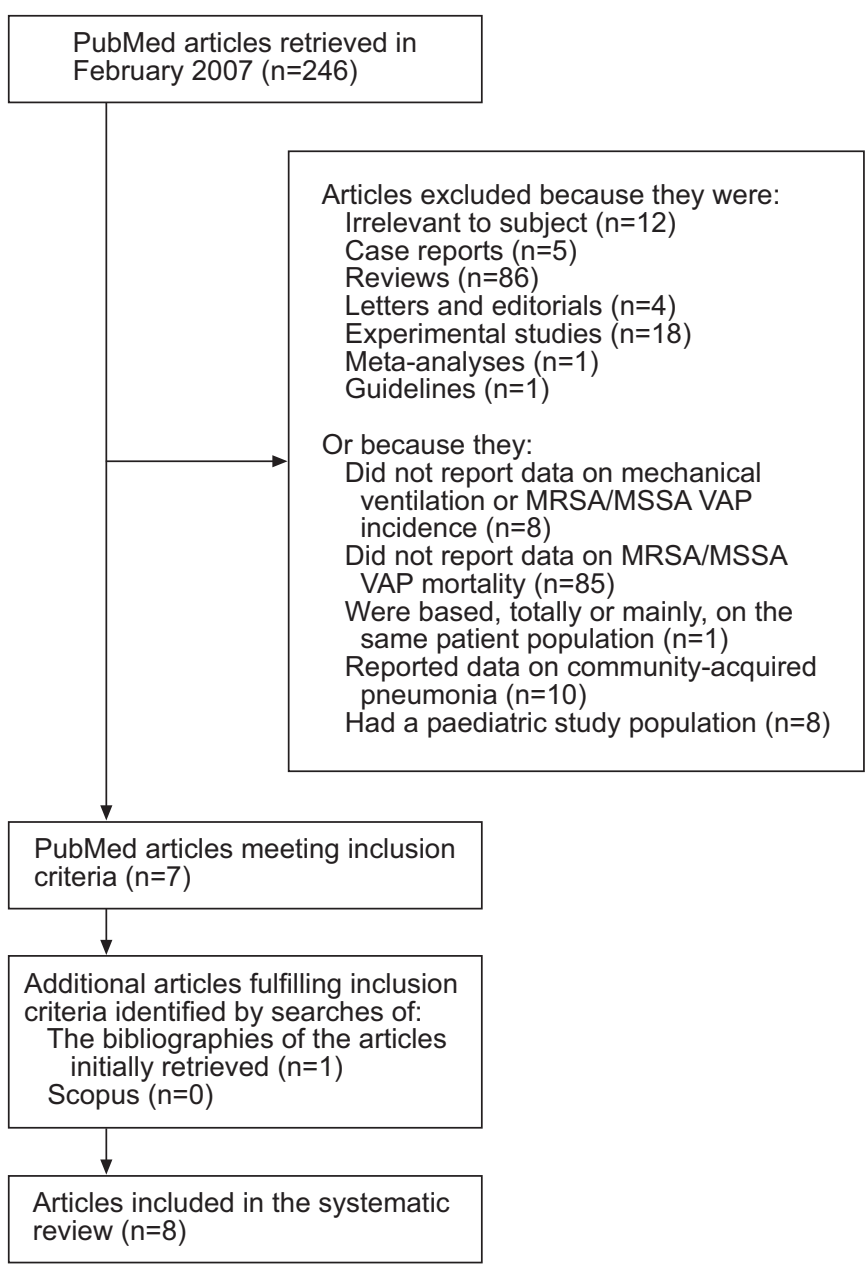

FIGURE 1. Flow diagram detailing reviewed articles and exclusion. MRSA: methicillin-resistant Staphylococcus aureus; MSSA: methicillin-sensitive Staphylococcus aureus; VAP: ventilator-associated pneumonia.

patients with MSSA VAP, patients with MRSA VAP were older in six studies $[14,18,19,21-23]$ and their ages did not differ in one [20]. The other study [24] did not give relevant data. The MRSA and MSSA groups were similar regarding sex in five $[14,18,19,22,23]$ of the eight eligible reports $[14,18-24]$ that provided such data.

Regarding the incidence of underlying diseases (namely cardiac, respiratory and neurological failure, as well as malignancy and diabetes mellitus) in the patients with pneumonia, data were available for six [14, 18-20,22, 23] of the eight eligible reports [14, 18-24]. In two studies [18, 23], cardiac [18] and respiratory failure [23] were more common among patients with MRSA (compared with MSSA) VAP, whereas, in two other reports [19, 22], neurological failure was more prominent in patients with MSSA (compared with MRSA) VAP. Severity of illness on admission was assessed in five [14, 18-20,22] of the eligible studies; no differences were shown between comparators. As opposed to patients with MSSA VAP, those with MRSA had a more prolonged duration of hospitalisation and mechanical ventilation in the included reports giving such data (table 2).

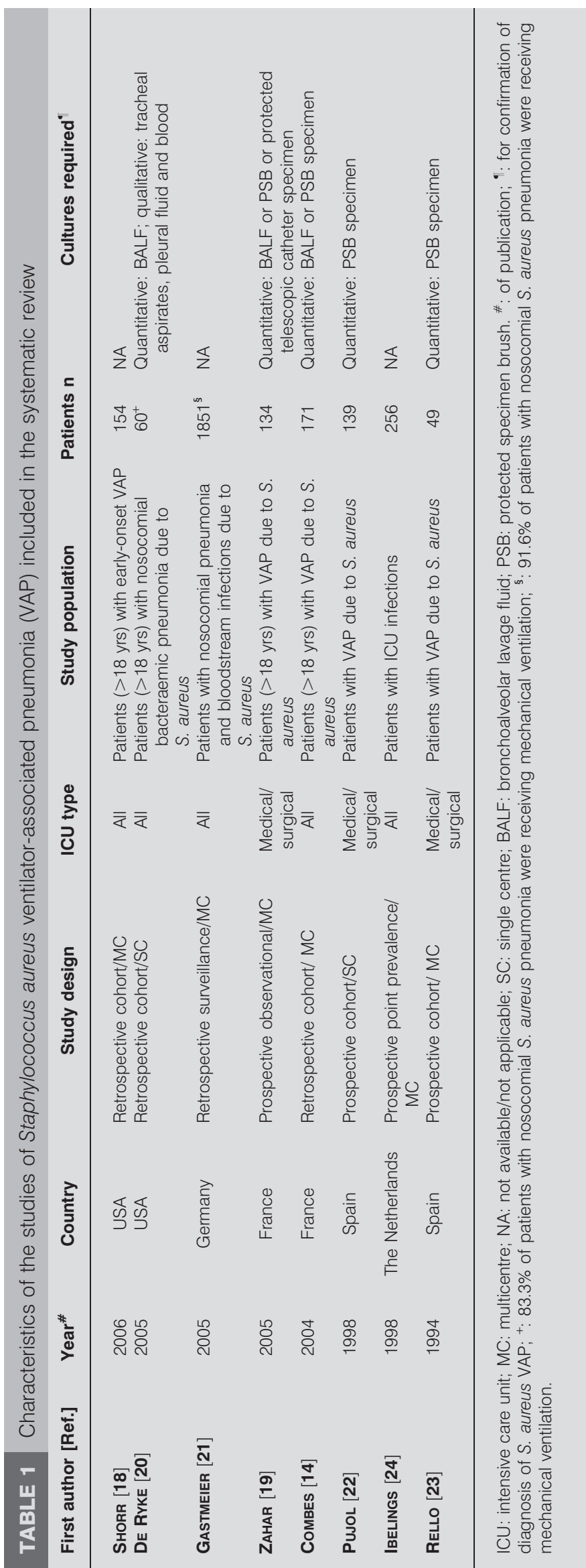




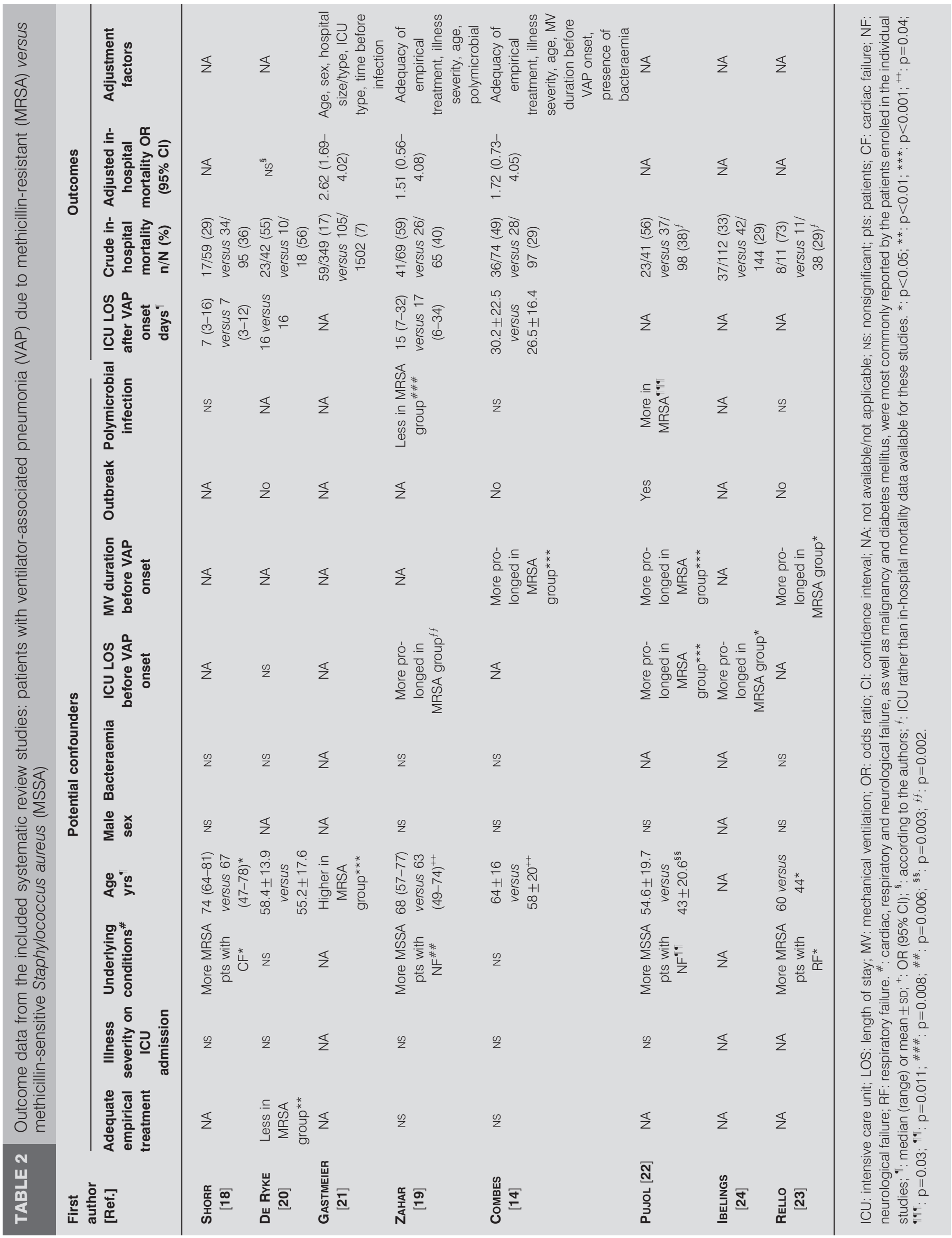




\section{Mortality}

The outcome data of the studies included in the systematic review are presented in table 2. Among 2,814 patients with S. aureus VAP, $757(27 \%)$ patients had MRSA VAP. With respect to the follow-up period for mortality, one [21] of the selected studies did not provide relevant information, whereas another [19] reported on both in-hospital and ICU mortality. In the remaining articles, this period was extended up to 6 weeks after VAP onset [24] or ICU discharge [22, 23] or hospital discharge $[14,18,20]$.

\section{Crude in-hospital and ICU mortality}

The crude in-hospital mortality was higher in patients with VAP due to MRSA as opposed to MSSA (OR 1.79 (95\% CI 1.210-2.65); data from eight reports [14, 18-24]; table 3 and fig. 2). This was also the case for crude ICU mortality (OR 2.49 (95\% CI 1.54-4.06); data from three reports [19, 22, 23]).

Subgroup analysis was performed after exclusion of the study that provided the largest number of patients, and that did not provide certain essential clinical (such as mortality during the follow-up period) and microbiological data (i.e. types of culture, if any, used for confirmation of the VAP diagnosis) [21]. Even after the exclusion of the above study, the finding of the main analysis did not change substantially (OR for crude in-hospital mortality 1.62 (95\% CI 1.06-2.48), with data from seven reports [14, 18-20, 22-24]).

\section{Adjusted in-hospital mortality}

In four [14, 19-21] of the eight reports included in the present review, mortality was adjusted for potential confounding factors, such as the adequacy of the empirical treatment [14, 19], severity of illness [14, 19], age [14, 19, 21], presence of bacteraemia [14] and duration of mechanical ventilation before [14] (table 2). One

\begin{tabular}{|c|c|c|c|c|}
\hline $\begin{array}{r}\text { TABLE } 3 \text { Ch } \\
\text { aur } \\
\text { inc }\end{array}$ & \multicolumn{3}{|c|}{$\begin{array}{l}\text { Characteristics of the studies of Staphylococcus } \\
\text { aureus ventilator-associated pneumonia } \\
\text { included in the systematic review }\end{array}$} & \multirow[t]{3}{*}{$\begin{array}{l}\text { lococcus } \\
\text { a }\end{array}$} \\
\hline \multirow[t]{2}{*}{ First author [Ref.] } & \multirow[t]{2}{*}{ Year ${ }^{\#}$} & \multicolumn{2}{|c|}{$\begin{array}{l}\text { Crude in-hospital mortality } \\
\qquad \mathrm{n} / \mathrm{N}\end{array}$} & \\
\hline & & MRSA & MSSA & \\
\hline SHORR [18] & 2006 & $17 / 59$ & $34 / 95$ & 12.91 \\
\hline De Ryke [20] & 2005 & $23 / 42$ & $10 / 18$ & 7.97 \\
\hline GAStMEIER [21] & 2005 & $59 / 349$ & $105 / 1502$ & 18.83 \\
\hline ZAHAR [19] & 2005 & $41 / 69$ & $26 / 65$ & 13.08 \\
\hline Combes [14] & 2004 & $36 / 74$ & $28 / 97$ & 13.99 \\
\hline Pujol [22] & 1998 & $23 / 41$ & $37 / 98$ & 12.35 \\
\hline IBELINGS [24] & 1998 & $37 / 112$ & $42 / 144$ & 15.65 \\
\hline Rello [23] & 1994 & $8 / 11$ & $11 / 38$ & 5.22 \\
\hline Overall & & $244 / 757$ & 293/2057 & 100 \\
\hline
\end{tabular}

[20] of these four reports did not give specific data regarding the confounders.

Of the four studies that were adjusted $[14,19,20,21]$, three [14, $19,20]$ failed to reveal a difference in in-hospital mortality between patients with MRSA and MSSA VAP (table 2). In contrast, in the remaining study [21], the mortality of patients with MRSA VAP remained higher than for those with MSSA VAP, even after adjustment (OR 2.62 (95\% CI 1.69-4.02)). However, it should be noted that the latter study [21] differed from the above in that it was adjusted for factors other than the adequacy of the initial treatment and severity of illness.

\section{Length of ICU stay after VAP onset}

Data on the length of ICU stay after VAP onset were available for four [14, 18-20] of the studies included in the present review. In three studies [14, 19, 20], there was no difference regarding length of stay between the compared groups, whereas, in the remaining study [18], length of ICU stay after VAP onset was more prolonged in the MRSA than in the MSSA group (table 2).

\section{DISCUSSION}

The findings of the present systematic review suggest that both the in-hospital and ICU mortality of patients with VAP due to $S$. aureus were higher in the presence than in the absence of methicillin resistance. Thus they are in line with those of a recent meta-analysis inferring that methicillin resistance is associated with increased mortality in patients with $S$. aureus bacteraemia [2].

However, the results of the current contribution contradict those of two clinical studies on the same issue that did not fulfil the present inclusion criteria [25, 26]. The fact that these two studies did not establish any association between methicillin resistance and excess mortality may not be surprising. Indeed, in the first study [25], $>50 \%$ of MSSA patients were treated with vancomycin (which was found to be associated with a higher mortality compared with cloxacillin [25]); this could account for their increased mortality and, thereby, the lack of difference between the MRSA and MSSA groups regarding this outcome. It is noteworthy that several investigators have reported that patients with MSSA infections experience worse clinical outcomes when treated with vancomycin rather than a $\beta$-lactam [27-29]. In the other study [26], several methodological issues (such as the enrolment of patients not receiving mechanical ventilation, as well as the absence of an accurate microbiological confirmation of the pneumonia diagnosis based on quantitative cultures) might have substantially influenced its findings. Likewise, three [18, $20,24]$ of the eight articles included in the present review also demonstrate no relationship between methicillin resistance and mortality in patients with $S$. aureus VAP. This may also be explained by their small sample size [20] and the considerable proportion of enrolled patients with MSSA nosocomial pneumonia who were treated with vancomycin [20], as well as the missing data regarding the duration of follow-up [18] and microbiological confirmation of S. aureus VAP $[18,24]$.

The mortality of patients with $S$. aureus VAP varied widely among the individual selected studies, a fact presumably explained by several considerations. For instance, there were 
First author [Ref.]

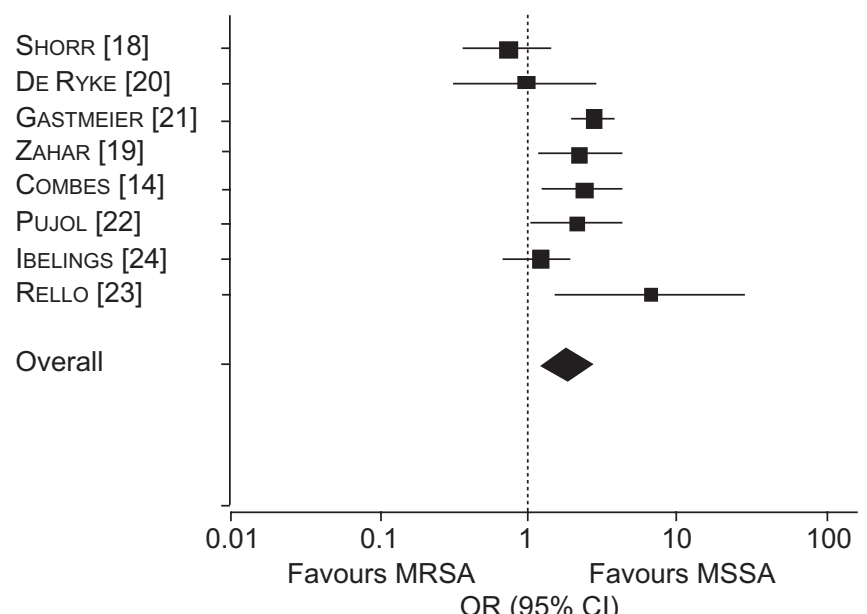

FIGURE 2. Forest plot comparing crude in-hospital mortality rates in studies of methicillin resistance versus sensitivity in patients with ventilator-associated pneumonia due to Staphylococcus aureus and the pooled analysis. Data are presented as odds ratio (OR; (size reflects weighting)) and 95\% confidence interval (Cl: horizontal bars). The centre of the diamond represents the pooled OR and its extremities the $\mathrm{Cl}$. ..... : line of no difference. If $\mathrm{OR}>1$, methicillin-sensitive S. aureus (MSSA) is favoured (i.e. mortality is lower); if $\mathrm{OR}<1$, methicillin-resistant $S$. aureus (MRSA) is favoured. In testing for heterogeneity of the pooled results that contributed towards the overall mean result, $\mathrm{X}^{2}=18.87$ (7 degrees of freedom; $p=0.009)$ and $\mathrm{I}^{2}=62.9 \%$. In testing for overall effect, $z=2.91 \quad(p=0.004)$.

differences in the mean age of patients enrolled in the selected studies. Given that age is an independent risk factor for mortality in patients with S. aureus VAP [14, 21], it seems plausible that studies enrolling young patients show low mortality. In addition, data, when available, dealing with the adequacy of empirical treatment and the severity of illness revealed substantial differences among the selected studies. It is also noteworthy that evaluation of the observed differences in severity of illness among the eligible reports could not be accurately performed, due to application of different disease severity indices. Finally, the follow-up periods were not identical among the selected reports; it might be argued that studies with more extended follow-up periods may also report higher mortality.

Another interesting finding of the present contribution is the possible attenuation of the effect of methicillin resistance on mortality when adjusting for adequacy of empirical treatment and severity of illness. It should be emphasised that this is based on only two reports [14, 19] including 305 patients. Even so, this finding may lead to several considerations. The fact that MRSA VAP causes increased mortality, compared with MSSA VAP, does not necessarily prove a causative relationship between methicillin resistance and excess lethality. It has previously been demonstrated that, compared with patients with MSSA bacteraemia, those with MRSA bacteraemia are more likely to be older, have a terminal disease and/or a more debilitated physical condition, require prolonged hospitalisation and mechanical ventilation, and receive inappropriate empirical treatment; it is interesting that, after adjustment for these confounding factors, methicillin resistance was not independently associated with excess mortality [30, 31]. Thus, it may be difficult to characterise MRSA as a more virulent pathogen than MSSA in terms of mortality, since its presence is associated with increased age [14, 19, 21], severity of illness [25] and inadequate treatment [6], all of which are independently associated with a worse prognosis.

In an attempt to explore the influence of numerous potential confounders on mortality, relevant information was collected from all of the selected studies. As depicted in table 2, in six $[14,18,19,21-23]$ of the eligible reports, patients with MRSA and MSSA differed in terms of age. However, in three [14, 19, 21] of these six studies [14, 18, 19, 21-23], this difference was resolved by performing a multivariable analysis. Furthermore, the evaluation of underlying diseases, a factor potentially influencing mortality, was performed in only six [14, 18-20, 22, 23] of the eligible reports. In addition, the assessment of severity of illness on admission and adequacy of empirical treatment was performed in only five [14, 18, 19, 20, 22] and three $[14,19,20]$ of the eligible studies, respectively. Conversely, duration of mechanical ventilation and length of stay before VAP onset were available for three $[14,22,23]$ and four reports [19, 20, 22, 24]; they were more prolonged in the MRSA than the MSSA groups. Parameters potentially influencing mortality other than the above, such as the presence of outbreak and polymicrobial status of VAP were assessed in four [14, 20, 22, 23] and five studies [14, 18, 19, 22, 23], respectively. These discrepancies in potential confounding factors presumably reflect substantial differences in patient population among eligible reports and cause difficulties in estimating the impact of methicillin resistance on mortality.

The present authors recognise that the current contribution has limitations. For example, the findings of the present systematic review may be influenced by the study that enrolled the largest number of patients with S. aureus VAP [21]. However, the main findings did not change substantially after exclusion of the above report. In addition, unfortunately, no data regarding the number of patients with early- or late-onset VAP in the MRSA and MSSA groups were provided by the great majority of the eligible reports. Finally, the heterogeneity among the included reports with regard to the factors for which there was adjustment, as well as the fact that, in half of the studies, there was absolutely no adjustment for confounding factors inevitably limit the value of the present evaluation of the available literature. However, in an attempt to investigate the influence of several confounding factors on mortality, relevant information was carefully gathered from all of the eligible reports (table 2); this probably permits the disclosure of possible associations between these factors and mortality.

In conclusion, despite the above mentioned limitations, the present authors believe that the current contribution is of clinical and health economic importance. The limited available and analysed evidence seems to suggest that methicillin resistance may be associated with excess mortality among persons who acquire Staphylococcus aureus ventilator-associated pneumonia, a finding that may differentiate the efforts to control methicillin-resistant Staphylococcus aureus. However, even more limited evidence exists suggesting that this association is not retained after adjustment for clinically significant potential confounding factors, and, thereby, that 
this association may not be causal but probably due to confounders, such as the adequacy of the empirical treatment and severity of illness.

\section{REFERENCES}

1 Barlow G, Nathwani D. Is antibiotic resistance a problem? A practical guide for hospital clinicians. Postgrad Med J 2005; 81: 680-692.

2 Cosgrove SE, Sakoulas G, Perencevich EN, Schwaber MJ, Karchmer AW, Carmeli Y. Comparison of mortality associated with methicillin-resistant and methicillin susceptible Staphylococcus aureus bacteremia: a meta-analysis. Clin Infect Dis 2003; 36: 53-59.

3 Travers K, Barza M. Morbidity of infections caused by antimicrobial-resistant bacteria. Clin Infect Dis 2002; 34: Suppl. 3, S131-S134.

4 Shurland S, Zhan M, Bradham DD, Roghmann MC. Comparison of mortality risk associated with bacteremia due to methicillin-resistant and methicillin-susceptible Staphylococcus aureus. Infect Control Hosp Epidemiol 2007; 28: 273-279.

5 Melzer M, Eykyn SJ, Gransden WR, Chinn S. Is methicillinresistant Staphylococcus aureus more virulent than methicillin-susceptible $S$. aureus? A comparative cohort study of British patients with nosocomial infection and bacteremia. Clin Infect Dis 2003; 37: 1453-1460.

6 Iregui M, Ward S, Sherman G, Fraser VJ, Kollef MH. Clinical importance of delays in the initiation of appropriate antibiotic treatment for ventilator-associated pneumonia. Chest 2002; 122: 262-268.

7 Harbarth S, Nobre V, Pittet D. Does antibiotic selection impact patient outcome? Clin Infect Dis 2007; 44: 87-93.

8 Schmitz FJ, Mac-Kenzie CR, Geisel R, et al. Enterotoxin and toxic shock syndrome toxin-1 production of methicillin resistant and methicillin sensitive Staphylococcus aureus strains. Eur J Epidemiol 1997; 13: 699-708.

9 Hershow RC, Khayr WF, Smith NL. A comparison of clinical virulence of nosocomially acquired methicillinresistant and methicillin-sensitive Staphylococcus aureus infections in a university hospital. Infect Control Hosp Epidemiol 1992; 13: 587-593.

10 Porzecanski I, Bowton DL. Diagnosis and treatment of ventilator-associated pneumonia. Chest 2006; 130: 597-604.

11 Chastre J, Fagon JY. Ventilator-associated pneumonia. Am J Respir Crit Care Med 2002; 165: 867-903.

12 Fridkin SK, Hill HA, Volkova NV, et al. Temporal changes in prevalence of antimicrobial resistance in 23 US hospitals. Emerg Infect Dis 2002; 8: 697-701.

13 Combes A, Luyt CE, Fagon JY, Wolff M, Trouillet JL, Chastre J. Early predictors for infection recurrence and death in patients with ventilator-associated pneumonia. Crit Care Med 2007; 35: 146-154.

14 Combes A, Luyt CE, Fagon JY, et al. Impact of methicillin resistance on outcome of Staphylococcus aureus ventilatorassociated pneumonia. Am J Respir Crit Care Med 2004; 170: 786-792.

15 Chastre J, Wolff M, Fagon JY, et al. Comparison of 8 versus 15 days of antibiotic therapy for ventilator-associated pneumonia in adults: a randomized trial. JAMA 2003; 290: 2588-2598.
16 Gonzalez C, Rubio M, Romero-Vivas J, Gonzalez M, Picazo JJ. Bacteremic pneumonia due to Staphylococcus aureus: a comparison of disease caused by methicillinresistant and methicillin-susceptible organisms. Clin Infect Dis 1999; 29: 1171-1177.

17 Theaker C, Ormond-Walshe S, Azadian B, Soni N. MRSA in the critically ill. J Hosp Infect 2001; 48: 98-102.

18 Shorr AF, Tabak YP, Gupta V, Johannes RS, Liu LZ, Kollef MH. Morbidity and cost burden of methicillinresistant Staphylococcus aureus in early onset ventilatorassociated pneumonia. Crit Care 2006; 10: R97.

19 Zahar JR, Clec'h C, Tafflet M, et al. Is methicillin resistance associated with a worse prognosis in Staphylococcus aureus ventilator-associated pneumonia? Clin Infect Dis 2005; 41: 1224-1231.

20 De Ryke CA, Lodise TP, Rybak MJ, McKinnon PS. Epidemiology, treatment and outcomes of nosocomial bacteremic Staphylococcus aureus pneumonia. Chest 2005; 128: 1414-1422.

21 Gastmeier P, Sohr D, Geffers C, Behnke M, Daschner F, Ruden H. Mortality risk factors with nosocomial Staphylococcus aureus infections in intensive care units: results from the German nosocomial infection surveillance system (KISS). Infection 2005; 33: 50-55.

22 Pujol M, Corbella X, Pena C, et al. Clinical and epidemiological findings in mechanically-ventilated patients with methicillin-resistant Staphylococcus aureus pneumonia. Eur J Clin Microbiol Infect Dis 1998; 17: 622-628.

23 Rello J, Torres A, Valles RJ, Gonzalez VJ, Artigas A, Rodriguez-Roisin R. Ventilator-associated pneumonia by Staphylococcus aureus. Comparison of methicillin-resistant and methicillin-sensitive episodes. Am J Respir Crit Care Med 1994; 150: 1545-1549.

24 Ibelings MMS, Bruining HA. Methicillin-resistant Staphylococcus aureus: acquisition and risk of death in patients in the intensive care unit. Eur J Surg 1998; 164: 411-418.

25 Gonzalez C, Rubio M, Romero-Vivas J, Gonzalea M, Picazo JJ. Bacteremic pneumonia due to Staphylococcus aureus: a comparison of disease caused by methicillinresistant and methicillin-susceptible organisms. Clin Infect Dis 1999; 29: 1171-1177.

26 Lentino JR, Hennein H, Krause S, et al. A comparison of pneumonia caused by gentamicin, methicillin-resistant and gentamicin, methicillin-sensitive Staphylococcus aureus: epidemiologic and clinical studies. Infect control 1985; 6: 267-272.

27 Stryjewski ME, Szczech LA, Benjamin DK Jr, et al. Use of vancomycin or first-generation cephalosporins for the treatment of hemodialysis-dependent patients with methicillin-susceptible Staphylococcus aureus bacteremia. Clin Infect Dis 2007; 44: 190-196.

28 Kopp BJ, Nix DE, Armstrong EP. Clinical and economic analysis of methicillin-susceptible and -resistant Staphylococcus aureus infections. Ann Pharmacother 2004; 38: 1377-1382.

29 Chang FY, Peacock JE Jr, Musher DM, et al. Staphylococcus aureus bacteremia: recurrence and the impact of antibiotic treatment in a prospective multicenter study. Medicine (Baltimore) 2003; 82: 333-339. 
30 Soriano A, Martines JA, Mensa J, et al. Pathogenic significance of methicillin resistance for patients with Staphylococcus aureus bacteremia. Clin Infect Dis 2000; 30: 368-373.
31 Harbarth S, Rutshmann O, Sudre P, Pitted D. Impact of methicillin resistance on the outcome of patients with bacteremia caused by Staphylococcus aureus. Arch Intern Med 1998; 158: 182-189. 